\title{
SPECTRA OF IRREDUCIBLE MATRICES
}

\author{
by HENRYK MINC $\dagger$
}

(Received 10th September 1973)

\section{Introduction}

A real matrix is called non-negative (positive) if all its entries are non-negative (positive). Two matrices $A$ and $B$ are said to be cogredient if there exists a permutation matrix $Q$ such that $Q A Q^{\Upsilon}=B$. A square non-negative matrix is called reducible if it is cogredient to a matrix of the form

$$
\left[\begin{array}{ll}
X & Z \\
0 & Y
\end{array}\right] \text {, }
$$

where the blocks $X$ and $Y$ are square. Otherwise it is called irreducible.

Frobenius (1) proved inter alia (see Section 3 below) that an irreducible matrix is cogredient to a matrix in the form

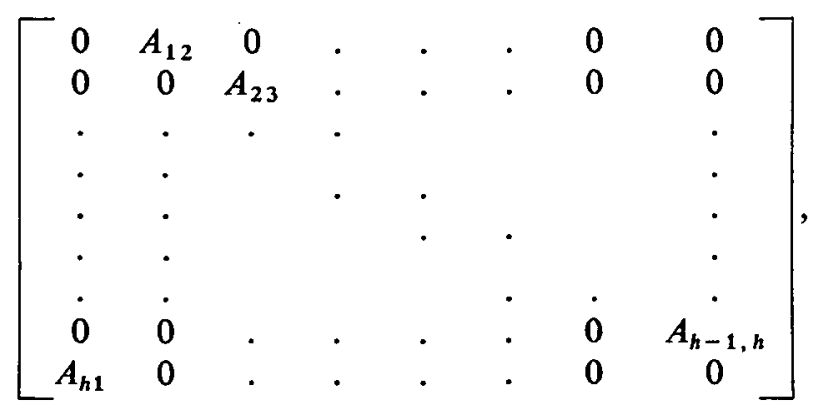

where the zero blocks along the main diagonal are square and $h$ is the index of imprimitivity of $A$, i.e. the number of eigenvalues of $A$ of maximal modulus (see Lemma 1 (c) in Section 3 below).

Mirsky (5) showed that if $A_{12}, A_{23}, \ldots, A_{h 1}$ are any complex $m$-square matrices (here $h$ is an arbitrary positive integer) and the eigenvalues of the product $A_{12} A_{23} \ldots A_{h 1}$ are $\omega_{1}, \ldots, \omega_{m}$, then the eigenvalues of the $h m$-square matrix in the form (1) with the $A_{i, i+1}$ in the indicated superdiagonal positions consist of all the $h$ th roots of $\omega_{1}, \ldots, \omega_{m}$ (a $h$ th root of zero being counted $h$ times).

In this paper I extend Mirsky's result to all complex matrices in the form (1) where the superdiagonal blocks $A_{12}, \ldots, A_{h 1}$ are not necessarily square, and I use this theorem to gain new information about the structure of irreducible matrices and their spectra.

$\dagger$ This research was supported by the Air Force Office of Scientific Research under Grant AFOSR-72-2164. 


\section{Main results}

Theorem 1. Let $A$ be an $n$-square complex matrix in the superdiagonal block form

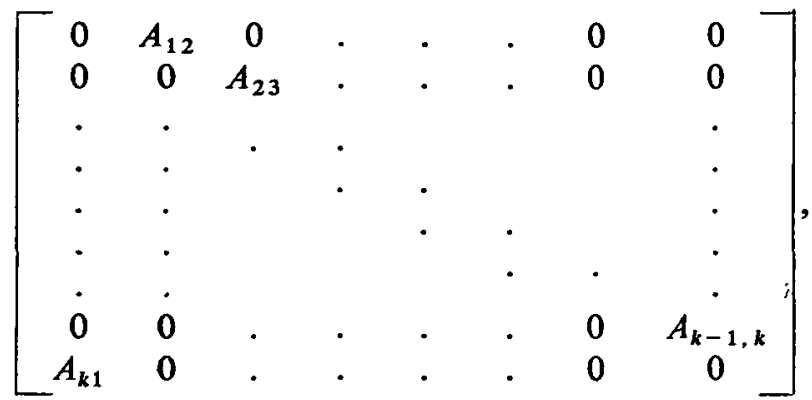

where the zero blocks along the main diagonal are square. Let $\omega_{1}, \ldots, \omega_{m}$ be the non-zero eigenvalues of the product $A_{12} A_{23} \ldots A_{k 1}$. Then the spectrum of $A$ consists of $n-k m$ zeros and the $k m k$ th roots of the numbers $\omega_{1}, \ldots, \omega_{m}$.

In order to exploit significantly Theorem 1 via the result of Frobenius to the case of irreducible non-negative matrices, we establish the following two auxiliary theorems which may be of interest in themselves.

Theorem 2. Let $B_{1}, \ldots, B_{s}$ and $C_{1}, \ldots, C_{t}$ be irreducible non-negative matrices. The direct sums

and

$$
G=\sum_{i=1}^{s} B_{i}
$$

$$
H=\sum_{i=1}^{t} C_{i}
$$

are cogredient if and only if $s=t$ and there exists a permutation $\sigma$ such that $B_{i}$ and $C_{\sigma(i)}$ are cogredient for $i=1, \ldots, s$.

Theorem 3. If $A$ is an irreducible non-negative matrix and if $A^{k}$ is cogredient to a direct sum of irreducible matrices $C_{1}, \ldots, C_{k}$, then $k$ divides the index of imprimitivity of $A$, and all the $C_{i}$ have the same non-zero eigenvalues.

By an application of the above theorems we obtain the following result.

Theorem 4. Let $A$ be an irreducible non-negative n-square matrix and suppose that $A^{k}$ is cogredient to a direct sum of irreducible matrices $C_{1}, \ldots, C_{k}$. If the nonzero eigenvalues of $C_{1}$ are $\omega_{1}, \ldots, \omega_{m}$, then the spectrum of $A$ consists of $n-k m$ zeros and the $k m k$ th roots of $\omega_{1}, \ldots, \omega_{m}$.

\section{Preliminaries}

Some known results are first stated for reference purposes.

Lemma 1 (Frobenius (1)). If $A$ is an irreducible non-negative matrix, then:

(a) $A$ has a real simple positive eigenvalue $r$ which is greater than or equal to the moduli of its other eigenvalues (the number $r$ is called the maximal eigenvalue of $A$ ); 
(b) there exists a positive eigenvector corresponding to $r$;

(c) if $A$ has $h$ eigenvalues of modulus $r$, then these are the distinct roots of $\lambda^{h}-r^{h}=0$ (the number $h$ is called the index of imprimitivity of $A$. If $h=1$, then $A$ is said to be primitive);

(d) $A$ is cogredient to a matrix in the form (1).

Lemma 2. If $A$ is a complex matrix in the form (2), then

$$
A^{k}=\sum_{t=1}^{k} B_{i}
$$

where $B_{t}=A_{t, t+1} A_{t+1, t+2} \ldots A_{t-1, t}, t=1, \ldots, k$.

Lemma 3 (Sylvester (6)). All the matrices $B_{t}$ defined in Lemma 2 have The same nonzero eigenvalues.

Lemma 4 (Minc (4)). Let $A$ be an irreducible non-negative matrix with index of imprimitivity $h$. Then $A$ is cogredient to a matrix in the form (2) with $k$ nonzero blocks in the superdiagonal if and only if $k$ divides $h$.

Lemma 5 (Minc (4)). If $A$ is an irreducible non-negative matrix in the form (2) with $k$ non-zero blocks in the superdiagonal, then

$$
A^{k}=\sum_{t=1}^{k} B_{t}
$$

where the blocks $B_{t}=A_{t, t+1} A_{t+1, t+2} \ldots A_{t-1, t}$ are irreducible.

The last auxiliary result is an extension to complex matrices of a theorem of Frobenius (1) on non-negative matrices.

Lemma 6. Let $A$ be a complex $n \times n$ matrix in the form (2), and let

$$
\lambda^{n}+\Sigma b_{\boldsymbol{t}} \lambda^{m_{t}},
$$

where the coefficients $b_{t}$ are non-zero, be the characteristic polynomial of $A$. Then $k$ divides $n-m_{t}$ for all $t$.

Proof of Lemma 6. Let $p(\lambda, M)$ denote the characteristic polynomial of $M$. Suppose that $A$ is in the form (2), where the block $A_{t, t+1}$ is $n_{t} \times n_{t+1}, t=1, \ldots$, $n-1$, and $A_{k 1}$ is $n_{k} \times n_{1}$, and let

$$
\begin{aligned}
& \text { where } \theta=\exp (2 \pi i / k) \text {. Then } D=\sum_{i=1}^{k} \theta^{t} I_{n_{t}}, \\
& \text { and therefore } D^{-1} A D=\theta A, \\
& \text { so that } \\
& \text { Hence } \\
& \qquad D^{-1}\left(\theta \lambda I_{n}-A\right) D=\theta\left(\lambda I_{n}-A\right), \\
& \quad p(\theta \lambda, A)=\theta^{n} p(\lambda, A) . \\
& \theta^{n} \lambda^{n}+\sum_{t} b_{t} \theta^{m_{t}} \lambda^{m_{t}}=\theta^{n} \lambda^{n}+\sum_{t} b_{t} \theta^{n} \lambda^{m_{t}},
\end{aligned}
$$

i.e.

$$
\theta^{m_{t}}=\theta^{n}
$$

E.M.S. $-19 / 3-Q$ 
for all $t$. Thus

for all $t$. The result follows.

$$
\exp \left(2 \pi i\left(n-m_{t}\right) / k\right)=1
$$

\section{Proofs}

Proof of Theorem 1. The proof is similar to that of Mirsky's theorem (5). By Lemma 3, the spectrum of $A^{k}$ consists of the numbers $\omega_{1}, \ldots, \omega_{m}$, each counted $k$ times, and $n-k m$ zeros. Thus

and therefore

$$
p\left(\lambda, A^{k}\right)=\lambda^{n-k m} \prod_{j=1}^{m}\left(\lambda-\omega_{j}\right)^{k}
$$

where

$$
p(\lambda, A)=\lambda^{n-k m} \phi(\lambda)
$$

$$
\phi(\lambda)=\sum_{t=1}^{k m} c_{t} \lambda^{t}
$$

By Lemma 6, a coefficient $c_{t}$ must vanish unless $k$ divides

$$
n-(n-k m+t)=k m-t \text {. }
$$

It follows that $c_{t}=0$ whenever $k$ does not divide $t$. In other words, $\phi(\lambda)$ is a polynomial in $\lambda^{k}$ :

for some numbers $\zeta_{1}, \ldots, \zeta_{m}$. Hence

$$
\phi(\lambda)=\prod_{t=1}^{m}\left(\lambda^{k}-\zeta_{t}\right)
$$

$$
\begin{aligned}
p(\lambda, A) & =\lambda^{n-k m} \prod_{t=1}^{m}\left(\lambda^{k}-\zeta_{t}\right) \\
& =\lambda^{n-k m} \prod_{1 \leqq j \leqq m}\left(\lambda-\zeta_{t}^{1 / k} \theta^{j}\right), \\
1 \leq j \leq k &
\end{aligned}
$$

where $\theta=\exp (2 \pi i / k)$ and $\zeta_{t}^{1 / k}$ denotes any fixed $k$ th root of $\zeta_{t}$. Therefore the characteristic polynomial of $A^{k}$ is

$$
p\left(\lambda, A^{k}\right)=\lambda^{n-k m} \prod_{t=1}^{m}\left(\lambda-\zeta_{t}\right)^{k}
$$

Comparing (3) and (5) it can be concluded that the numbers $\zeta_{1}, \ldots, \zeta_{m}$ are the same as the numbers $\omega_{1}, \ldots, \omega_{m}$, in some order. Thus the characteristic equation (4) of $A$ reads

and the theorem is established.

$$
p(\lambda, A)=\lambda^{n-k m} \prod_{\substack{1 \leqq \\ 1 \leqq j \\ j \leqq k}}\left(\lambda-\omega_{t}^{1 / k} \theta^{j}\right),
$$

Proof of Theorem 2. The sufficiency of the conditions is quite obvious. To prove the necessity let $P$ be a permutation matrix such that

$$
P^{\top} G P=H
$$


and let $\tau$ be the permutation corresponding to $P$, so that the $(i, j)$ entry of $G$ is permuted into the $(\tau(i), \tau(j))$ position of $H=P^{\top} G P$. For brevity the notation $\bar{i}$ is used in place of $\tau(i)$. Denote by $A\left[\mu_{1}, \ldots, \mu_{a} \mid v_{1}, \ldots, v_{b}\right]$ the submatrix of $A$ lying in rows numbered $\mu_{1}, \ldots, \mu_{a}$ and columns numbered $v_{1}, \ldots, v_{b}$; the rows $\mu_{1}, \ldots, \mu_{a}$ of $A$ (and the columns $v_{1}, \ldots, v_{b}$ ) are said to intersect the submatrix. Now suppose that for some $v, 1 \leqq v \leqq \mathrm{t}$,

$$
C_{v}=H\left[\bar{\alpha}_{1}, \ldots, \bar{\alpha}_{p}, \bar{\beta}_{p+1}, \ldots, \bar{\beta}_{q} \mid \bar{\alpha}_{1}, \ldots, \bar{\alpha}_{p}, \bar{\beta}_{p+1}, \ldots, \bar{\beta}_{q}\right] \text {, }
$$

and that rows and columns $\alpha_{1}, \ldots, \alpha_{p}$ of $G$ intersect block $B_{u}$ but none of rows nor columns $\beta_{p+1}, \ldots, \beta_{q}$ of $G$ intersect $B_{u}$. However, the only non-zero entries in the rows $\alpha_{1}, \ldots, \alpha_{p}$ of $G$ are in the columns $\alpha_{1}, \ldots, \alpha_{p}$. Thus

and therefore

$$
G\left[\alpha_{1}, \ldots, \alpha_{p} \mid \beta_{p+1}, \ldots, \beta_{q}\right]=0,
$$

$$
H\left[\bar{\alpha}_{1}, \ldots, \bar{\alpha}_{p} \mid \bar{\beta}_{p+1}, \ldots, \bar{\beta}_{q}\right]=0 .
$$

But this would imply that $C_{v}$ is reducible. Hence the supposition is impossible, and each of the $C_{j}$ can intersect only rows and columns corresponding to rows and columns that intersect a single $B_{i}$. Since $\sum_{i=1}^{t} C_{i}$ and $\sum_{i=1}^{s} B_{i}$ are cogredient, the result follows.

Proof of Theorem 3. It is first shown that $k$ must divide the index of imprimitivity $h$ of $A$. Let $r$ be the maximal eigenvalue of $A$ and let $x$ be a positive eigenvector corresponding to $r$. Then $x$ is an eigenvector of $A^{k}$ corresponding to $r^{k}$. Now, $A^{k}$ is cogredient to $\sum_{t=1}^{k} C_{t}$ and therefore $r^{k}$ is an eigenvalue (clearly of maximal modulus) of each $C_{t}$. Since the $C_{t}$ are irreducible, the eigenvalue $r^{k}$ is simple and therefore $A^{k}$ has exactly $k$ eigenvalues equal to $r^{k}$. But Lemma 1 (c) implies that there are $d=\operatorname{gcd}(h, k)$ such eigenvalues. Hence $d=k$ and thus $k$ divides $h$.

It now follows from Lemma 4 in conjunction with Lemma 2 and Lemma 3 that $A^{k}$ is cogredient to

$$
\sum_{t=1}^{k} B_{t}
$$

where the $B_{t}$ are irreducible and all the $B_{t}$ have the same non-zero eigenvalues. But then $\sum_{t=1}^{k} B_{t}$ and $\sum_{t=1}^{k} C_{t}$ are cogredient, and all the $B_{t}$ and all the $C_{t}$ are irreducible. Thus by Theorem 2 the $B_{1}, \ldots, B_{k}$ are cogredient to the $C_{1}, \ldots, C_{k}$, in some order, and the result follows.

Proof of Theorem 4. By Theorem 3, $k$ divides the index of imprimitivity of $A$, and thus by Lemma 4, the matrix $A$ is cogredient to a matrix in the form (2) with blocks $A_{12}, A_{23}, \ldots, A_{k 1}$ in the superdiagonal. Then $A^{k}$ is cogredient to $\sum_{t=1}^{k} B_{t}$, where $B_{t}=A_{t, t+1} A_{t+1, t+2} \ldots A_{t-1, t}, t=1, \ldots, k$, and all the $B_{t}$ have 
the same non-zero eigenvalues. Hence by Theorem 2 and Theorem 3, the matrices $B_{1}$ and $C_{1}$ have the same non-zero eigenvalues. The result now follows by virtue of Theorem 1 .

\section{REFERENCES}

(1) G. Frobenius, Über Matrizen aus nicht negativen Elementen, S.-B. Deutsch. Akad. Wiss. Berlin Math.-Nat. Kl. (1912), 456-477.

(2) F. R. Gantmacher, The Theory of Matrices, vol. II (Chelsea Publishing Company, New York, 1959).

(3) H. Minc, Irreducible matrices, Linear and Multilinear Algebra 1 (1974), 337-342.

(4) H. Minc, The structure of irreducible matrices, Linear and Multilinear Algebra 2 (1974), 85-90.

(5) L. MirsKy, An inequality for characteristic roots and singular values of complex matrices, Monatsh. Math. 70 (1966), 357-359.

(6) J. J. SyLVESTER, On the equation to the secular inequalities in the planetary theory, Philos. Mag. (5) 16 (1883), 267-269.

INSTITUTE FOR ALGEBRA AND COMBINATORICS

UNIVERSITY OF CALIFORNIA

SANTA BARBARA, 93106, U.S.A. 